\title{
Potência de Larmor de uma partícula carregada submetida a um campo elétrico gerado por dois discos dielétricos homogeneamente carregados
}

Larmor's power of a charged particle under the effect of an electric field generated by two homogeneously charged dielectric disks

\author{
Ellen Carolinie Gomes e Silva*1@ ${ }^{*}$, Yony Walter Gonzales ${ }^{2}$ \\ ${ }^{1}$ Universidade Federal de Santa Catarina, Trindade, Florianópolis, SC, Brasil. \\ ${ }^{2}$ Universidade Federal do Amapá, Macapá, AP, Brasil.
}

\begin{abstract}
Recebido em 26 de setembro de 2020. Revisado em 29 de outubro de 2020. Aceito em 31 de outubro de 2020.
Neste artigo, investigamos a dinâmica de uma partícula carregada quando submetida a um campo elétrico gerado por dois discos dielétricos e homogêneos. Analisamos a construção da equação de movimento para a carga acelerada, que na aproximação linear da força elétrica resulta num Movimento Harmônico Simples (MHS). Posteriormente, a força de radiação de Abraham-Lorentz é incorporada na equação de movimento devido o movimento acelerado da carga resultar na emissão de radiação de energia e, nessa situação, se comporta como um Oscilador Harmônico Amortecido (OHA). E calculamos a potência de radiação de Larmor para esse movimento amortecido da partícula.
\end{abstract}

Palavras-chave: Oscilador Harmônico Simples, Oscilador Harmônico Amortecido, Força de Abraham-Lorentz, Cálculo de radiação.

\begin{abstract}
In this article, we investigate the dynamics of a charged particle when subjected to an electric field generated by two dielectric and homogeneous disks. We analyzed the construction of the motion equation for the accelerated charge, which in the linear approximation of the electric force results in a Simple Harmonic Oscillator (SHO). Subsequently, the Abraham-Lorentz radiation force is incorporated into the motion equation because the accelerated movement of the load results in the emission of energy radiation and, in this situation, behaves like a Damped Harmonic Oscillator (DHO). And we calculated Larmor's radiation power for this damped particle motion.
\end{abstract}

Keywords: Simple Harmonic Oscillator, Damped Harmonic Oscillator, Abraham-Lorentz force, Calculation of radiation.

\section{Introdução}

O comportamento de uma carga elétrica acelerada é estudado pela eletrodinâmica clássica quando a força que a acelera é proveniente da interação com campos eletromagnéticos externos. O movimento acelerado da carga nesses campos externos inclui necessariamente a emissão de radiação por parte da carga. Dito isso, a radiação emitida carrega energia, momento linear e momento angular, influenciando num tempo posterior o movimento da carga. Assim, o movimento da carga elétrica estará determinado, em parte, pela forma de emissão da radiação [1, 2].

De fato, na eletrodinâmica clássica a radiação de um sistema de cargas pode ser calculada quando se conhece o movimento delas, já que isso é suficiente para determinar os campos que aparecem no vetor de Poynting. Hendrik Lorentz (1853-1928) demonstrou que

\footnotetext{
* Endereço de correspondência: ellencar811@gmail.com
}

o campo nas proximidades de uma carga com simetria esférica é distorcido pelos efeitos combinados entre a aceleração dessa carga e da velocidade de propagação finita das alterações do campo; tal distorção gera uma "autoforça" resultante do campo sobre a carga [3]. O deslocamento dessa força pode representar, em certos casos, a energia eletromagnética radiada. Assim, Lorentz conclui que a energia de radiação de uma carga é puramente cinética quando a carga é acelerada por um campo eletromagnético externo em um sistema de coordenadas inercial 3 .

Em 1897, em um artigo intitulado "On theory of the magnetic Influence on spectra and the radiation from moving ions", Joseph Larmor apresentou uma demonstração de que partículas carregadas e aceleradas emitiam ondas eletromagnéticas, obtendo a fórmula para calcular essa potência de energia irradiada, o que ficou conhecida como a fórmula da potência de radiação de Larmor 4]. A referida fórmula foi inicialmente utilizada para calcular a intensidade das linhas espectrais do 
átomo do hidrogênio. Posteriormente, em 1898, Liénard apresenta seu trabalho "Champ électrique et magnétique produit par une charge électrique contentre é en un point et animée d'un mouvement quelconque", onde demonstração que a fórmula de Larmor somente era válida para partículas carregadas em movimento não relativístico [4].

Do ponto de vista da dinâmica de uma partícula, Abraham e Lorentz deduziram - a partir da fórmula de Larmor -, a força que estava relacionada a emissão de radiação da carga para o caso não relativístico é chamada de Força de Radiação de Abraham-Lorentz, essa força teria que ser incorporada na equação de movimento para uma partícula carregada submetida a um campo eletromagnético externo. A generalização para o caso relativístico é chamada de Força de Radiação de Abraham-Lorentz-Dirac.

Neste trabalho foi utilizada a força de AbrahamLorentz no estudo do movimento de uma carga pontual submetida a um campo elétrico gerado por dois discos dielétricos de raios $R$, bem como uma distribuição de cargas homogêneas em relação a um sistema de referência inercial, desconsiderando a força gravitacional. Trata-se da delimitação de um estudo que objetivou a compreensão da aplicabilidade dos polinômios de Legendre para calcular o potencial elétrico fora do eixo de simetria axial entre dois discos dielétricos, e que desconsiderou a dissipação de radiação da carga acelerada, e, portanto, a força de Abraham-Lorentz não foi incorporada na equação de movimento [5].

\section{Desenvolvimento do Modelo Matemático}

\subsection{Força eletrostática dos discos sobre a carga pontual}

Consideremos um sistema que consta de dois discos dielétricos paralelos de raios iguais a $R$, com densidade uniforme de carga $\sigma$ e separados por uma distância $2 L$. No eixo de simetria é colocado uma carga pontual $q$ de mesmo sinal dos discos, como se mostra na Figura 1

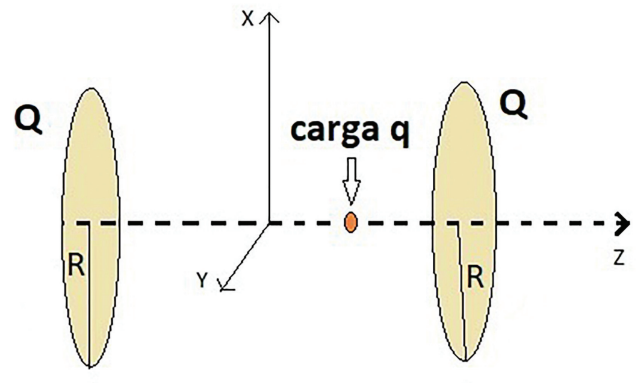

$2 \mathrm{~L}$

Figura 1: Representação esquemática do problema. Fonte: Elaborada pelos autores (2020)

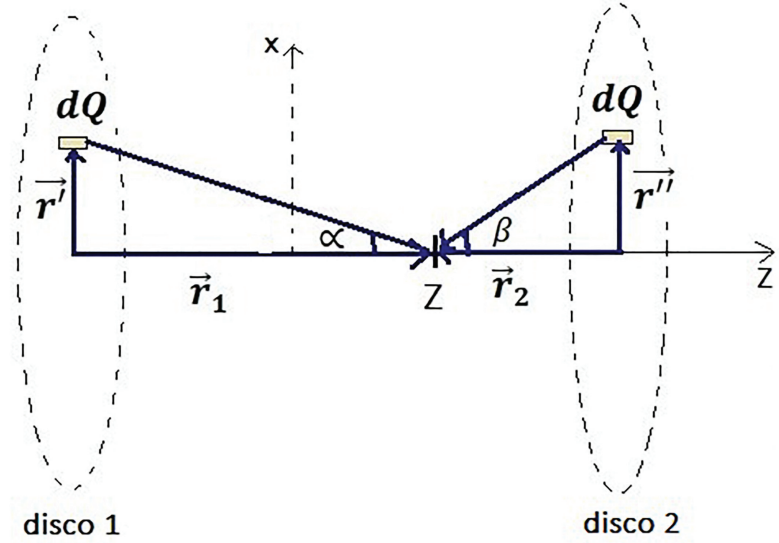

Figura 2: Detalhes vetoriais da representação esquemática. Fonte: Elaborada pelos autores (2020).

A força eletrostática dos discos é calculada em uma posição $Z$ localizada entre eles ao longo do eixo de simetria $z$, definimos o referencial e os vetores necessários para este fim (ver Figura 22). A densidade de carga dos discos é dada por:

$$
\sigma=\frac{Q}{A}=\frac{d Q}{d A}=\frac{d Q}{r^{\prime} d r^{\prime} d \theta}
$$

Segundo o referencial da Figura 2, as quantidades definidas para o cálculo do campo elétrico são dadas por [5]:

a) Disco 1:

$$
\begin{gathered}
\vec{r}_{1}=(L+Z) \hat{z} \\
\vec{r}^{\prime}=r^{\prime} \cos \theta \hat{x}+r^{\prime} \operatorname{sen} \theta \hat{y} \\
\vec{r}_{1}-\vec{r}^{\prime}=(L+Z) \hat{z}-r^{\prime} \cos \theta \hat{x}-r^{\prime} \operatorname{sen} \theta \hat{y}
\end{gathered}
$$

Assim,

$$
\begin{aligned}
\left|\vec{r}_{1}-\vec{r}^{\prime}\right| & =\sqrt{(L+Z)^{2}+r^{\prime 2} \cos ^{2} \theta+r^{\prime 2} \operatorname{sen}^{2} \theta} \\
& =\sqrt{(L+Z)^{2}+r^{\prime 2}}
\end{aligned}
$$

$\mathrm{E} \cos \alpha=\frac{(L+Z)}{\sqrt{(L+Z)^{2}+r^{\prime 2}}}$.

Levando em conta a simetria do problema, o campo elétrico do disco 1 é ao longo do eixo $z$, se anulando nos eixos $x$ e $y$, assim temos que:

$\vec{E}_{1}=\frac{\sigma}{4 \pi \varepsilon_{0}} \int_{0}^{R} \int_{0}^{2 \pi} \frac{r^{\prime} d r^{\prime} d \theta}{\left[(L+Z)^{2}+r^{\prime 2}\right]} \cdot \frac{(L+Z)}{\sqrt{(L+Z)^{2}+r^{\prime 2}}} \hat{z}$

$\mathrm{O}$ que resulta em:

$$
\vec{E}_{1}=\frac{\sigma}{2 \varepsilon_{0}}\left[1-\frac{(L+Z)}{\sqrt{(L+Z)^{2}+R^{2}}}\right] \hat{z}
$$

\section{b) Disco 2:}

Procedendo de forma análoga temos que:

$$
\begin{gathered}
\vec{r}_{2}=-(L-Z) \hat{z} \\
\vec{r}^{\prime \prime}=r^{\prime \prime} \cos \theta \hat{x}+r^{\prime \prime} \operatorname{sen} \theta \hat{y}
\end{gathered}
$$


Assim:

$$
\begin{aligned}
\left|\vec{r}_{2}-\vec{r}^{\prime \prime}\right| & =\sqrt{(L-Z)^{2}+r^{\prime \prime 2} \operatorname{sen}^{2} \theta+r^{\prime \prime} \cos ^{2} \theta} \\
& =\sqrt{(L-Z)^{2}+r^{\prime \prime 2}}
\end{aligned}
$$

$\mathrm{E} \cos \beta=\frac{(L-Z)}{\sqrt{(L-Z)^{2}+r^{\prime \prime 2}}}$

Logo o campo elétrico do disco 2 é calculado da mesma forma o que resulta em:

$$
\vec{E}_{2}=-\frac{\sigma}{2 \varepsilon_{0}}\left[1-\frac{(L-Z)}{\sqrt{(L-Z)^{2}+R^{2}}}\right] \hat{z}
$$

A força eletrostática dos dois discos sobre a carga pontual $q$ é dada pela superposição dos campos elétricos determinados, isto é:

$$
\begin{gathered}
\vec{F}_{Z}=q\left(\vec{E}_{1}+\vec{E}_{2}\right) \\
\vec{F}_{Z}=\frac{\sigma q}{2 \varepsilon_{0}}\left[\frac{(L-Z)}{\sqrt{(L-Z)^{2}-R^{2}}}-\frac{(L+Z)}{\sqrt{(L+Z)^{2}-R^{2}}}\right] \hat{z}
\end{gathered}
$$

Ou:

$$
\begin{aligned}
\vec{F}_{Z}= & \frac{\sigma q}{2 \varepsilon_{0}}\left[\frac{(L-Z)}{R} \frac{1}{\sqrt{1+\left(\frac{L-Z}{R}\right)^{2}}}\right. \\
& \left.-\frac{(L+Z)}{R} \frac{1}{\sqrt{1+\left(\frac{L+Z}{R}\right)^{2}}}\right] \hat{z}
\end{aligned}
$$

Se a separação $2 L$ entre os discos é menor que o raio $R$, então temos que $\frac{(L-Z)}{R}<1$ e $\frac{(L+Z)}{R}<1$, o que devemos considerar que $Z \ll L$. Com isso, as expressões das raízes podem ser expandidas em série de Taylor:

$$
\begin{aligned}
\vec{F}_{Z}= & \frac{\sigma q}{2 \varepsilon_{0}}\left[\frac{(L-Z)}{R}\left(1-\frac{1}{2} \frac{(L-Z)^{2}}{R^{2}}+\cdots\right)\right. \\
& \left.-\frac{(L+Z)}{R}\left(1-\frac{1}{2} \frac{(L+Z)^{2}}{R^{2}}+\cdots\right)\right] \hat{z}
\end{aligned}
$$

Considerando as condições geométricas impostas acima, a componente resultante da força eletrostática na direção do eixo $z$ na aproximação linear de $Z$ exercida pelos discos sobre a carga pontual $q$ fica:

$$
\vec{F}_{Z}=\frac{\sigma q}{2 \varepsilon_{0} R}\left(\frac{3 L^{2}}{R^{2}}-2\right) Z \hat{z}
$$

\subsection{Equação de movimento na aproximação linear}

Do resultado anterior da força sobre a carga $q$, escrevemos a equação diferencial resultante da segunda lei de Newton para o movimento da carga que resulta em:

$$
\ddot{Z}+\frac{\sigma q}{m R \varepsilon_{0}}\left(\frac{3 L^{2}}{R^{2}}-2\right) Z=0
$$

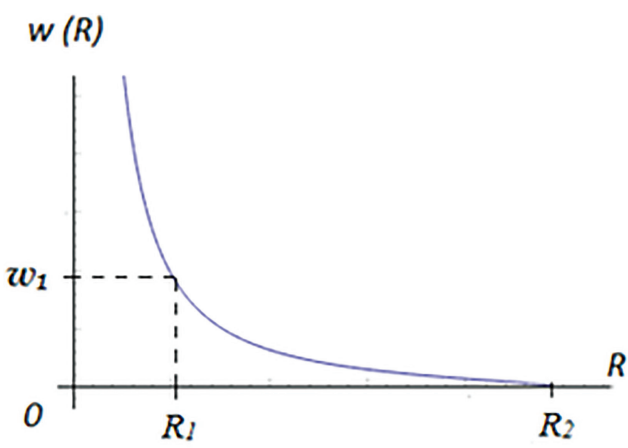

Figura 3: Comportamento da frequência de oscilação $\omega$ dada pela equação (3) da carga $q$ em função do raio $R$ dos discos para um dado valor $L$, onde $R_{1}=L, R_{2}=\sqrt{3 / 2} L$ e $\omega_{1}=$ $\sqrt{\frac{\sigma q}{m \varepsilon_{0} L}}$. Fonte: Elaborado pelos autores (2020) por meio do WolframAlpha ${ }^{1}$

A equação 2 mostra que a carga pontual pode realizar movimentos do tipo oscilador harmônico simples ao longo do eixo de simetria $z$, cuja frequência de oscilação aproximada é dada por:

$$
\omega=\sqrt{\frac{\sigma q}{m R \varepsilon_{0}}\left(\frac{3 L^{2}}{R^{2}}-2\right)}
$$

A equação (3) mostra que a frequência de oscilação da carga depende da relação dos parâmetros geométricos entre a distância de separação $L$ e o raio $R$ dos discos. Assim, para que a carga $q$ possa realizar pequenas oscilações harmônicas, a configuração do sistema deve satisfazer a condição de que $L \leq R \leq \sqrt{\frac{3}{2}} L$, e consequentemente, os valores da frequência $\omega$ ficarão restritos à $0<\omega<\sqrt{\frac{\sigma q}{m \varepsilon_{0} L}}$. A Figura 3 representa a dependência da frequência de oscilação em função do raio dos discos para um dado valor de $L$.

Por fim, a solução da equação (2) é dada por:

$$
Z(t)=A \cos (\omega t+\emptyset)
$$

Onde a amplitude $A$ e a diferença de fase $\emptyset$ são definidas pelas condições iniciais do problema.

\subsection{A força dissipativa de Abraham-Lorentz}

No problema estudado, a força elétrica exercida pelos discos sobre a carga $q$ gera um movimento do tipo oscilador harmônico, portanto essa carga irradiará (dissipará) energia, como foi demonstrado por Larmor [1]. Para levar em conta a situação física mais real na equação de movimento da carga $q$, incorporamos a força de radiação que se adequa a esta situação não relativística e que pode ser derivada da potência de radiação de Larmor, isto é,

\footnotetext{
1 Ferramenta computacional disponível em https://www.wol framalpha.com/examples/mathematics/calculus-and-analysis/
} 
a força de dissipação de Abraham-Lorentz 1]. Para o nosso caso é dada da seguinte forma:

$$
\vec{F}_{\text {rad }}=\frac{q^{2}}{6 \pi \varepsilon_{0} c^{3}} \dddot{\vec{Z}} \quad \text { ou } \quad \vec{F}_{\text {rad }}=\beta \ddot{\vec{Z}}
$$

Onde $\beta=\frac{q^{2}}{6 \pi \varepsilon_{0} c^{3}}, \varepsilon_{0}$ é a permissividade do vácuo e $c$ a velocidade da luz. Assim, a força de Abraham-Lorentz é proporcional à derivada da aceleração da carga.

Da equação (4), calculamos que:

$$
\begin{gathered}
\dot{Z}=-A \omega \sin (\omega t+\emptyset) ; \quad \ddot{Z}=-A \omega^{2} \cos (\omega t+\emptyset) ; \\
\dddot{Z}=-\omega^{2} \dot{Z}
\end{gathered}
$$

Com isso, escrevemos a força de radiação na forma:

$$
F_{\text {rad }}=-\beta \omega^{2} \dot{Z}
$$

No nosso caso $Z$ é a posição na solução harmônica simples para o movimento da carga $q$. Assim, escrevemos a equação diferencial resultante da segunda lei de Newton para o movimento da carga considerando tanto a força eletrostática dos dois discos quanto a força de radiação em regime não relativístico, temos:

$$
m \ddot{Z}=F_{Z}+F_{\text {rad }}
$$

O que resulta na equação diferencial para o movimento da carga $q$ :

$$
\ddot{Z}+\gamma \omega^{2} \dot{Z}+\omega^{2} Z=0
$$

A equação (5) representa a equação de movimento de um oscilador harmônico linearmente amortecido. Observe que o termo dissipativo desta equação vem calibrado pela frequência oscilação $\omega$ e a constante $\gamma=\beta / m$. A solução dessa equação diferencial é bem conhecida e tem a forma geral:

$$
Z=C e^{b t}
$$

Substituindo-o na equação (5) obtemos a equação característica:

$$
b^{2}+\gamma \omega^{2} b+\omega^{2}=0
$$

As raízes obtidas são $b=-\frac{\gamma \omega^{2}}{2} \pm\left[\frac{\gamma \omega^{2}}{4}-\omega^{2}\right]^{1 / 2}$, pelo qual se pode ter três situações diferentes: (a) $\frac{\gamma \omega^{2}}{4}<\omega^{2}$, (b) $\frac{\gamma \omega^{2}}{4}>\omega^{2}$ e (c) $\frac{\gamma \omega^{2}}{4}=\omega^{2}$.

Aqui somente vamos considerar o caso (a) que corresponde ao regime de movimento com amortecimento subcrítico da carga, nesse caso trabalhamos com as raízes:

$$
b=-\frac{\gamma \omega^{2}}{2} \pm i \Omega
$$

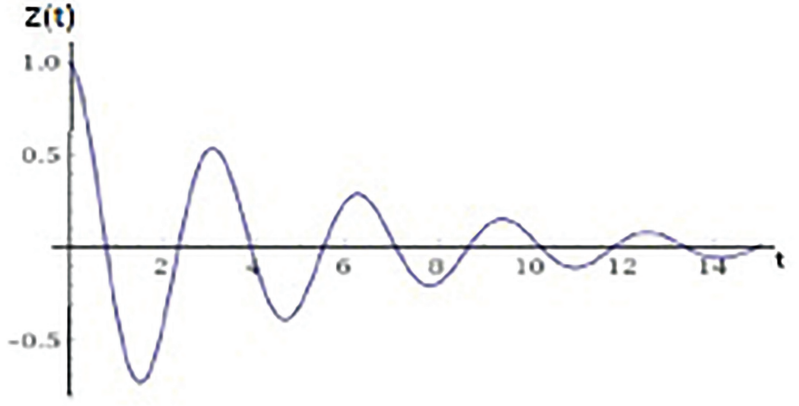

Figura 4: Decaimento da amplitude da carga oscilante em função do tempo dado pela equação $(9)$ com valor $\mathrm{A}=1$. Fonte: Elaborado pelos autores (2020) por meio do WolframAlpha.

Onde $\Omega=\frac{\omega}{2} \sqrt{4-\gamma^{2} \omega^{2}}$ é a frequência de amortecimento subcrítico, logo a solução geral para este caso é dada pela combinação linear das soluções com cada uma das raízes, isto é:

$$
Z=Z_{+} e^{-\frac{\gamma \omega^{2}}{2} t+i \Omega t}+Z_{-} e^{-\frac{\gamma \omega^{2}}{2} t-i \Omega t}
$$

E utilizando as funções trigonométricas escrevemos a solução de forma mais compacta:

$$
Z=A e^{-\frac{\gamma \omega^{2}}{2} t} \cos (\Omega t+\theta)
$$

Onde o valor de $A$ e $\theta$ dependem das condições iniciais estabelecidas para a carga $q$, que no nosso caso é colocada, inicialmente, em repouso na posição $Z_{0}$ localizada no eixo entre os discos. Assim as constantes são dadas por $A=\frac{Z_{0}}{\cos \theta}$ e $\tan \theta=\frac{\gamma \omega^{2}}{2 \Omega}$.

Por outro lado, considerando a frequência de amortecimento subcrítico $\Omega$ temos que $4-\gamma^{2} \omega^{2}>0$, portanto, a frequência de oscilação, para este caso, fica estabelecida como:

$$
\omega^{2}=\frac{\sigma q}{m R \varepsilon_{0}}\left(\frac{3 L^{2}}{R^{2}}-2\right)<\frac{4}{\gamma^{2}}
$$

Um comportamento qualitativo da solução subcrítica para o movimento da carga é mostrado na Figura 4

\section{Potência de Radiação de Larmor}

A potência de radiação de Larmor para o movimento de uma carga $q$ acelerada é dada por 1

$$
P=\frac{q^{2}}{6 \pi \varepsilon_{0} c^{3}} a^{2}=\beta a^{2}
$$

Onde $a$ é o módulo da aceleração da carga, neste caso $a=\ddot{Z}$, sendo $Z$ a solução da posição da carga no regime subcrítico amortecido que estamos considerando. Utilizando essa situação temos que:

$$
Z=A e^{-\frac{\gamma \omega^{2}}{2} t} \cos (\Omega t+\theta)
$$


Logo a velocidade será:

$\dot{Z}=-\frac{A \gamma \omega^{2}}{2} e^{-\frac{\gamma \omega^{2}}{2} t} \cos (\Omega t-\theta)-A \Omega e^{-\frac{\gamma \omega^{2}}{2} t} \sin (\Omega t-\theta)$

E a aceleração, então, fica:

$$
\begin{aligned}
\ddot{Z}= & A e^{-\frac{\gamma \omega^{2}}{2} t}\left[\left(\frac{\gamma^{2} \omega^{2}}{4}-\Omega^{2}\right) \cos (\Omega t+\theta)\right. \\
& \left.+\Omega \gamma \omega^{2} \sin (\Omega t+\theta)\right]
\end{aligned}
$$

Daqui podemos escrever uma equação mais compacta para a aceleração considerando as seguintes relações trigonométricas:

$$
\sin \Phi=\frac{\left(\frac{\gamma^{2} \omega^{4}}{4}-\Omega^{2}\right)}{\sqrt{\left(\frac{\gamma^{2} \omega^{4}}{4}-\Omega^{2}\right)^{2}+\left(\Omega \gamma \omega^{2}\right)^{2}}}
$$

e

$$
\cos \Phi=\frac{\Omega \gamma \omega^{2}}{\sqrt{\left(\frac{\gamma^{2} \omega^{4}}{4}-\Omega^{2}\right)^{2}+\left(\Omega \gamma \omega^{2}\right)^{2}}}
$$

O que resulta em:

$$
\ddot{Z}=A e^{-\frac{\gamma \omega^{2}}{2} t} \sqrt{\left(\frac{\gamma^{2} \omega^{4}}{4}-\Omega^{2}\right)^{2}+\left(\Omega \gamma \omega^{2}\right)^{2}} \sin (\Omega t+\alpha)
$$

Onde $\alpha=\theta+\Phi$. Assim, da equação (11) na equação 10, a potência de Larmor para o movimento amortecido subcrítico da carga $q$ resulta em:

$$
\begin{gathered}
P=\beta A^{2}\left(\left(\frac{\gamma^{2} \omega^{4}}{4}-\Omega^{2}\right)^{2}+\left(\Omega \gamma \omega^{2}\right)^{2}\right) \\
e^{-\gamma \omega^{2} t} \sin ^{2}(\Omega t+\alpha)
\end{gathered}
$$

Colocando a expressão de $\Omega$ na equação 12 esta é simplificada resultando finalmente em:

$$
P=\beta A^{2} \omega^{2} e^{-\gamma \omega^{2} t} \sin ^{2}\left(\frac{\omega}{2} \sqrt{4-\gamma^{2} \omega^{2}} t+\alpha\right)
$$

Onde a frequência $\omega$ é dada pela equação (3). A Figura 5 mostra qualitativamente o comportamento da potência de radiação de Larmor em função do tempo dada pela equação 13 .

Como se observa na Figura 5, a amplitude da potência de radiação de Larmor para a partícula decai de forma exponencial tendendo a zero quando a partícula alcança o repouso no ponto de equilíbrio. As amplitudes da potência podem ser modificadas alterando os valores dos parâmetros na equação (13) dentro das restrições estabelecidos para o movimento amortecido subcrítico.

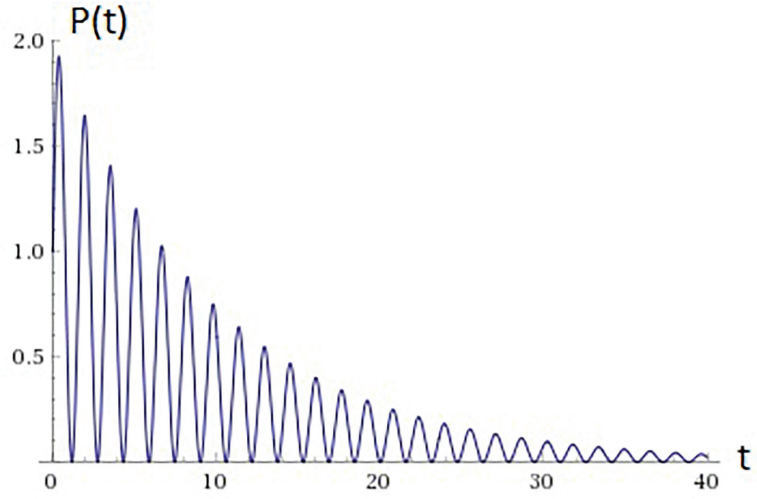

Figura 5: Representação gráfica para a potência de radiação de Larmor em função do tempo para o movimento oscilatório da carga $q$, no regime subcrítico amortecido. Fonte: Elaborado pelos autores (2020) por meio do WolframAlpha.

\section{Considerações Finais}

Foi estudada a dinâmica de uma partícula carregada submetida a uma força elétrica gerada por dois discos dielétricos carregados homogeneamente e de mesmo sinal que a partícula. Impondo a condição de pequenas oscilações e a configuração geométrica estabelecida, encontramos que a carga $q$ oscila com frequência harmônica aproximada $\omega$ que depende da relação $\left(\frac{L}{R}\right)$. A análise do movimento ocorreu na aproximação linear dada pela força elétrica que depende linearmente de $Z$. Ao fixar um valor para $L$, encontramos que essa frequência harmônica somente pode acontecer para valores onde $L<R<\sqrt{\frac{3}{2}} L$.

Como toda carga em movimento acelerado por um campo eletromagnético irradia energia, então foi possível introduzir uma força de radiação na equação de movimento da carga, que no caso não relativístico e a nível da eletrodinâmica clássica é a força de radiação de Abraham- Lorentz, resultando que a dinâmica que descreve o movimento da carga é semelhante ao movimento de uma partícula que se move como um oscilador harmônico amortecido. Foram analisadas as condições físicas e restrições geométricas onde esse regime subcrítico acontece.

Finalmente, a potência de radiação de Larmor foi calculada e o resultado é mostrado na equação 13 . A expressão da potência de Larmor obtida é representada pelo produto de duas funções temporais, basicamente de um seno quadrático envolvida por uma queda exponencial, onde a amplitude e a frequência de amortecimento subcrítico estão parametrizadas pela frequência $\omega\left(\frac{L}{R}\right)$ e as constantes físicas representadas por $\gamma$.

\section{Referências}

[1] M. Phillips e W.K.H. Panofsky, Classical Electricity and Magnetism (Addison-Welsey Publishing Company, United States of America, 1962). 
[2] J.D. Jackson, Classical Electrodynamics (John Wiley and Sons, United States of America, 1999).

[3] R.P. Feynam, R.B. Leighton e M. Sands, Lições de física de Feynman: a edição definitiva (Bookman, Porto Alegre, 2008).

[4] D.P.M. Filho e J.K.S. Kamassury, Revista Brasileira de Ensino de Física 40, 3 (2018).

[5] E.C.G. e Silva, Oscilações de uma partícula carregada num campo elétrico produzido por dois discos uniformemente carregados. Trabalho de Conclusão de Curso, Universidade Federal do Amapá, Amapá (2016). 